\title{
35. STELLAR CONSTITUTION (CONSTITUTION DES ÉTOILES)
}

\author{
PRESIDENT: A. Maeder (Switzerland) \\ VICE-PRESIDENT: P. Demarque (USA) \\ SECRETARY: Y. Lebreton (France)
}

24 July 1991

\section{BUSINESS MEETING}

The meeting starts by a moment of silence with the members standing in respect for three members of the Commission who passed away in the last three years. They were T.G. Cowling, President of the Commission from 1952 to 1958, P. Ledoux, President of the Commission from 1964 to 1967, and J. Trominen.

Commission 35 has democratically chosen the new Officers according to a procedure initiated after the Montreal General Assembly. Firstly, a call for candidatures was made in January 1990 and then a ballot was performed in March 1990 to designate a new Vice President, who in principle at the next term becomes President, and new members of the Organising Committee, to replace the former members having served for 6 years. The poll was satisfactory, $35 \%$ of the Commission members participated in the vote. For the term 1991-1994, the elected President is Pierre Demarque (USA) and the Vice President Cesare Chiosi (Italy). The members of the Organising Committee are: R. Canal, I. Then, A. Maeder, G. Michaud, K. Nomoto, A. Renzini, A.V. Tutukov, D. VandenBerg and G. Vauclair. The President, Vice-President and the members of the Organising Committee were endorsed by the Executive Committee at the General Assembly.

The Commission Report with contributions of F. D'Antona, P. Demarque, Y. Totsuka, E.L. Fitzpatrick, S. Kwok, J.C. Wheeler, E.P.J. van den Heuvel and G. Meynet was sent to all Commission members. In answer to questions the General Secretary addressed to Commission Presidents, clear statements were expressed at the business meeting in favour of the continuation of this form of Commission Report.

During the last triennium, 3 circular letters were sent to all Commission members and 14 to the Committee members. The President mentions that there had been a great increase in the number of requests for sponsorship of scientific meetings. Each member of the Organising Committee has been consulted. The general philosophy is that we are not to approve anything, but to help and support valuable meetings, since the Executive Committee needs pertinent advices to make a choice among numerous proposals. In this way, our Commission has given sponsorship to IAU colloquia or symposia. The titles are 1) Nonlinear phenomena in stellar variability, 2) Planetary nebulae, 3) The cosmic dynamo, 4) The bulges of galaxies, 5) Inside the stars, 6) New perspectives on stellar pulsation and pulsating variable stars.

The President closes the business meeting by some general considerations on the present status of the field of Stellar Constitution set into the general astronomical context. He notes that this domain remains, and is going to remain, at the crossroads of most research lines in astrophysics. The observational input keeps going to be very large; just to mention SN 1987A, the X and gamma bursts, the solar and stellar oscillations, the data from high resolution spectroscopy and the observations of stars in external galaxies. All these areas need large developments in theoretical models and important investments on physical ingredients. This is the essence of the field of Stellar Constitution, which presently experiences such an 
impressive expansion, that the domain can no longer be mastered by a single researcher. In that respect, Stellar Constitution is meeting just what Science experienced a few centuries back and what Astronomy encountered a few decades ago.

\section{SCIENTIFIC MEETINGS}

Commission 35 held a total of 10 scientific sessions, in addition to the business meeting. Two sessions were devoted to "Evolution of Stars and Star Clusters in the Magellanic Clouds" and are summarized below. One session was a "Progress Report on Opacities, Equation of State and their Effects on Solar and Stellar Models" and is also summarized below. Two sessions were held with Commission 34 on "Star Formation and the Interstellar Medium", see Report of Commission 34; one session on "Progress in Helioseismology" was held together with Commissions 10 and 12, see Report of Commission 10 and 12. Four sessions were organized on "Early Nucleosynthesis in Galaxies" with Commissions 28 and 29; due to space requirements, only the titles of the contributions are given below. A session on the "Progenitor Evolution of SN 1987A" had unfortunately to be cancelled due to the fire in the Conference Center.

Commission 35 also co-sponsored a Joint Discussion on Origin of Stars and Planetary Systems and two Joint Commission Meetings on Atomic and Molecular Data for Space Astronomy and on Late Evolution of Low Mass Stars. Proceedings will be found in Highlights of Astronomy.

\section{July 1991}

\section{Evolution of stars and star clusters in the Magellanic Clouds}

Chaired by C. Chiosi and D. Sugimoto
M. Mateo
C. Chiosi

A. Maeder

S.Van den Bergh

G. Meylan

D. Sugimoto

N. R. Walborn

A. Ray,

N. Ratlinasree

M. Kigushi, S. Narita

and C. Hayashi
Constraints on the IMF from Magellanic Cloud star clusters

Confrontation of stellar evolution theory with observation of Magellanic Cloud clusters (summary not received)

WR and $\mathrm{O}$ stars in the Magellanic Clouds

Age distribution of clusters in the LMC and SMC

Observational constraints on the dynamics of Magellanic Clouds globular clusters

Dynamical evolution of single and binary globular clusters

Two-stage starbursts in the LMC

Evolution of massive binary stars in the LMC and its implication for pulsar population

Stability of a nearly Keplerian disk embedded in corona

Mateo reviewed photometric determinations of the IMF for stars between 1-10 $M_{\odot}$ in Magellanic Cloud star clusters. The earliest studies by Mateo, Elson et al. yielded very different results, although there were at that time no clusters in common to both samples. Recent CCD studies suggest that crucial differences in methodology can account for the earlier discrepant results. Specifically, the Elson et al. photographic results appear to be erroneous: recent CCD studies of some of the clusters in their sample consistently yield much steeper slopes. Differences in the results derived from various CCD studies are systematically correlated with the manner in which completeness corrections are determined and applied. Current studies show that the observed slopes of the IMF of Magellanic Clond clusters range from 1.0 to 2.5 (the Salpeter value being 1.35), with a mean value near 1.8. Because of the systematic differences among various studies, the actual range in IMF slopes is surely smaller than this; a single, universal IMF 
slope near 1.8 may be applicable to most clusters. The present results provide no evidence for significant variations in the IMF slope correlated with age or metallicity.

Maeder discussed WR and $O$ stars distributions in the Magellanic Clouds. Among the stellar properties showing very large differences from galaxy to galaxy, the number frequencies of WR stars and the number ratios of WN/WC stars are extreme cases. In the solar neighbourhood, the LMC and SMC the number ratios of $\mathrm{WR} / \mathrm{O}$ stars are respectively $0.12,0.04,0.015$; for the $\mathrm{WC} / \mathrm{WN}$ ratios, the values are $1.9,0.26$, 0.14 . The studies by Maeder show that the origin of these differences mainly lies in the stellar evolution. For massive stars, differences in metallicity $\mathrm{Z}$ mainly act through stellar winds, which according to recent wind models are higher at larger metallicities. Thus, a larger $Z$ implies more peeling off and more WR stars formed, with higher WC/WN ratios. Quantitative comparisons between the Maeder stellar models and observations in the Galaxy, the LMC, the SMC and other galaxies very well support this view. The link Z-mass-loss-evolution has also a great impact on the nucleosynthesis and chemical yields.

Van den Bergh reviewed evidence on the rates of star and cluster formation in the Magellanic Clouds. In the SMC star formation seems to have turned on gradually with the oldest cluster (NGC121) having an age of about $12 \mathrm{Gyr}$. In the LMC an initial burst of activity about $15 \mathrm{Gyr}$ ago formed a number of massive globular clusters and numerous field RR Lyrae stars. After this the large clond remained quiescent for about 10 Gyr. A second burst of star and cluster formation started 3-4 Gyr ago and continues to the present day. The fact that star and cluster formation rates in the LMC and SMC are not correlated proves that such bursts of star formation did not result from gravitational interactions between the clouds. The fact that clusters with ages of 5-10 Gyr are seen in the SMC shows that their absence in the LMC is NOT due to selective effects.

Meylan reviewed the problems related to the dynamics of globular clusters. Because of the large spread in age among clusters, the Magellanic Clouds provide a unique opportunity to learn about cluster formation and evolution. Dynamical models (e.g. King-Michie) need three observational constraints: 1) the surface brightness profile gives an indication on the concentration. From a survey of Magellanic clusters, it appears that, among old clusters, the fraction of collapsed to King-Michie type clusters is the same in the Galaxy and in the LMC. 2) the color-magnitude diagram gives a precise determination of the cluster age when compared with stellar models, for NGC 2070, comparison with isochrones from Maeder and Meynet (1991) gives an age smaller than $3 \times 10^{6} \mathrm{yr}$. Contrary to Melnick (1985), no turnoff is observed in the Meylan et al. data which concern only the Melnick's field. This could be an indication of delayed star formation in the central parts of the cluster. The luminosity function is derived from the color-magnitude diagram stars; for NGC 2070 an IMF exponant $x=0.7 \pm 0.4$ is found. 3) the velocity dispersion is obtained from integrated light spectra (Dubath et al. 1990). Combined with the surface brightness profile, the velocity dispersion constraints a King-Michie dynamical model and allows determination of mass and mass-luminosity ratio. Core velocity dispersions have been obtained for 23 galactic clusters, 28 LMC clusters, and 3 clusters of the Fornax dwarf spheroidal. All the old globular clusters studies in the Galaxy, the LMC and Fornax display strong similitudes in the ranges for velocity dispersion, mass, and $\mathrm{M} / \mathrm{Lv}$ ratio, indicating a possible universality of their characteristics and formation mode.

Main objectives of Sugimoto's talk is to extend cooperations between the two fields, stellar evolution and stellar dynarnics. The gravothermal catastrophe and suceeding post-collapse evolution with binary hardening of globular cluster are the same physical processes as the gravitational contraction and the nuclear burning of a single star, respectively. Evolution and merging of binary globular clusters, which are observed in LMC, are quite similar to Roche lobe overflow and coalescence of close binary stars. Formation of binary stars and millisecond pulsars in the core of globular clusters are one of the common subjects. Differences in chemical evolution among LMC, SMC and the Galaxy as discussed in the preceeding talks should also be interpreted under both lights of stellar evolution and the dynamical history of LMC-SMCGalaxy system. Special purpose computer being developed for $\mathrm{N}$-body problem is also applicable to smoothed hydrodynamics of stars.

Walborn reviewed two-stage starbursts in the LMC. A study of the stellar content of the LMC giant shell H II region N11 by Parker, Garmany, Massey, and Walborn has revealed significant differences between the concentrated association occupying the central, evacuated cavity and those ionizing the surrounding nebulosities. The former contains an earliest spectral type of $06, \mathrm{OB}$ supergiants, and a WC + late-O 
system, implying an age of $510^{6} \mathrm{yr}$, while the latter contains (newly discovered) 03 stars, candidate ZAMS $O$ stars, and have a flatter IMF, consistent with an age of $310^{6} \mathrm{yr}$. This morphology is remarkably reminiscent of that in 30 Doradus, where IR protostars (age less than $10^{6} \mathrm{yr}$ ) have recently been found surrounding the central 3-million-year-old cluster. Thus N11 appears as an aged 30 Dor; its central $\mathrm{WC}+\mathrm{O}$ system may even be an evolved R136. Furthermore, N11, which is the second-ranked H II region in the LMC, is located diametrically opposite 30 Dor, off the other end of the Bar. A hypothesis emerges whereby the initial, centrally concentrated starbursts are caused by events on a galactic scale, and winds/supernovae from its massive stars trigger a secondary burst around the periphery about $210^{6}$ yr later. The two mechanisms may produce different IMFs.

The LMC Hertzsprung-Russel diagram compiled by Fitzpatrick and Garmany shows supergiants immediatly redwards of the main sequence while theoretical models of massive stars with normal hydrogen abundance predict that this region should be unpopulated. Supergiants which have accreted helium from a binary companion, further evolve in a way so that the models and observed data are consistent (Tuchman and Wheeler). Ray and Rathnasree compare the optical data on OB supergiants with models of massive stars with a LMC metallicity with and without He-enriched envelopes and conclude that about $60 \%$ of supergiants may occur in binaries. These binaries will evolve into massive X-ray binaries, the observed number and orbital period distribution of which constrain the scenarios of supergiant binaries evolution. The distribution of post main sequence binaries and related systems like $\mathrm{WR}+\mathrm{O}$ stars so obtained is bimodal with close and wide binaries in which the latter type is dominating. The expected space velocity distribution of runaway $\mathrm{O}$-stars arising from the first explosion in the binary system are calculated. The second Supernova disrupts the binary giving rise to two runaway neutron stars. The steady-state spin and radio luminosity distributions of single pulsars born from the massive stars is determined under simple assumptions. A small but significant number of observable single radio pulsars arising out of the disrupted massive binaries may appear in the few millisecond spin period range. Most pulsars coming from long period binaries will have a low velocity of ejection and might cluster around the $\mathrm{OB}$ associations in the LMC.

Kigushi, Narita and Hayashi have carried out a numerical simulation for the time evolution of a protosolar nebula enclosed by a corona. The initial condition for the calculation is a equilibrium configuration of the type of $f(r) \cdot g(\theta)$. For the configuration used the density is 300 times greater in the equator than the pole, the temperature is 30 times greater in the pole than the equator, and the thickness of the disk is $\delta \theta$ about 0.15 . This configuration is stable when the adiabatic condition is imposed. However if temperature is assumed to be a function of position, turbulence develops just above the disk and penetrates into the disk. The resulting disk accretion parameter $\alpha$ is greater than about 0.2 in the disk, which is a marginal value below which Roche instability develops. This shows the importance of the radiative transfer for the estimate of the angular momentum transfer in the disk.

\section{$\underline{27 \text { July } 1991}$}

Progress report on opacities, equation of state and their effects on solar and stellar models Chaired by A. Maeder

W. Däppen

A.N. Cox

D. Guenther
Present status of the opacity project

Opacity calculations, Cepheids and OB star instabilities

Input physics and effects on solar models and oscillations

Däppen gave information on the present status of new opacity calculations. Currently, there are two large projects to compute solar and stellar opacities, one being the international so-called "Opacity Project (OP)" led by Seaton and Mihalas, the other an effort pursued at Livermore by Rogers and Iglesias, called "OPAL". While OP is based on very detailed ab-initio atomic physics, it relies on a more heuristic description of the plasma. OPAL is based on a systematic treatment of the quantum statistical mechanics of the plasma, but it uses more simplified atomic physics (parametric potentials). At a recent meeting held in Caracas, these two groups compared results, and it emerged that both achieved significant progress by 
yielding a factor of 2-3 enhancement of the heavy-element opacity (compared with the older Los Alamos opacities), while agreeing with each other to $10-20 \%$. Preliminary calculations have shown that these new opacities can indeed solve the problem with the mass of beat and bump Cepheids. It looks therefore that after some 25 years of great successes with Los Alamos astrophysical opacities a new era has begun, and promising applications of both OP and OPAL data are on the way.

Cox shows that recent stellar (OPAL) opacities from the Lawrence Livermore National Library have been very successful in explaining problems in stellar structure and stability. OPAL include numerous improvements in all the basic photon absorption processes, but their most dramatic effect is the inclusion of the millions of same-shell formally forbidden iron lines. These $\mathrm{M}$ shell transitions give the most lines and the most increase at a temperature centered around $250,000 \mathrm{~K}$ at a density of near $10^{-5} \mathrm{~g} \mathrm{~cm}^{-3}$. These iron lines increase the opacity for yellow giants envelopes by a factor of three typically. Such opacity increases have been shown to change the yellow giant pulsator envelope structures to decrease their apparent density concentration for the pulsation modes so that the period ratio between the first radial overtone and the fundamental mode decreases enough to agree well with that observed using traditional mass models. These larger opacities also can deepen the convection zone during pre-main sequence lithium burning for stars now seen as Li deficient G giants in the Hyades. The most impressive effect of the new larger and more rapidly rising opacities with temperature is the large enough kappa effect in OB stars envelopes so that the beta Cephei pulsations can be predicted. This prediction of pulsational instability now depends on the iron abundance, and stable stars can exist if there is not enough iron. To explain variability in all kinds of $O B$ stars (including Be stars, luminous blue variables, and even very hot helium deficient stars) and their episodes of constancy and variability, apparently levitation of iron in the pulsation driving layers by photon momentum absorption is required. A small but sufficient iron concentration increase can initiate pulsations, but they then might cause enough shear to remix the envelope to a stable condition again. The $O B$ star pulsation problems have now been reduced to composition problems in the layers about $10^{-6}$ of the mass into the star.

Guenther presented several solar models each of them differing in the input physics adopted (table 1) and compared them to observations. In conclusion, with respect to improving the p-modes, the most important new physics are: low-T opacities, T-tau relation for atmosphere, equation of state's effect on thermodynamic variables. None of these affect the interior structure. With respect to changing the solar interior, the most important new physics are: high- $\mathrm{T}$ opacities and $\mathrm{P}$, rho, $\mathrm{T}$ changes via Coulomb corrections in equation of state. All solar models have an initial helium abundance $Y$ of about 0.28 . The OPAL opacities deepen the solar convection zone to a depth consistent with p-mode inversions. The neutrino flux is not significantly changed.

Table 1: Model characteristics

\begin{tabular}{|lccccccc|}
\hline Model & $\mathrm{Y}$ & $\alpha$ & $\log \mathrm{T}_{c}$ & $\log \rho_{c}$ & $\mathrm{X}$ conv & $\mathrm{M}$ conv/ $M_{\odot}$ & $\log \mathrm{T}$ conv \\
\hline Standard model & 0.2875 & 1.195 & 7.186 & 2.166 & 0.745 & 0.0153 & 6.284 \\
MHD equ. of state & 0.2736 & 1.256 & 7.188 & 2.159 & 0.734 & 0.0190 & 6.308 \\
D.-H. correction & 0.2771 & 1.223 & 7.185 & 2.160 & 0.740 & 0.0167 & 6.294 \\
Cox low T opacities & 0.2876 & 2.066 & 7.186 & 2.166 & 0.744 & 0.0153 & 6.285 \\
Kurucz low T opac. & 0.2875 & 1.559 & 7.186 & 2.166 & 0.745 & 0.0152 & 6.283 \\
OPAL opacities & 0.2918 & 1.248 & 7.193 & 2.168 & 0.724 & 0.0211 & 6.331 \\
Ross-Aller mixture & 0.2843 & 1.242 & 7.190 & 2.166 & 0.738 & 0.0177 & 6.299 \\
Bahcall nuclear rates & 0.2843 & 1.194 & 7.189 & 2.181 & 0.744 & 0.0151 & 6.284 \\
K. S. atmosphere & 0.2875 & 1.423 & 7.186 & 2.166 & 0.745 & 0.0155 & 6.284 \\
\hline
\end{tabular}


Early nucleosynthesis in galaxies

Chaired by P. Conti and A. Maeder
P. Conti
Early nucleosynthesis - Nature of the problem
D. Lambert Pop II stars: CNO abundances and light metals
C. Pilaichowski
Abundances in old clusters
M. Bessel
Stellar composition in the Magellanic clouds
H. Lamers
J. Bergeron
D. Hollowell
Abundances of low-mass post AGB stars
A. Maeder
Abundances in absorption line systems towards QSOs
AGB star model at low Z
B. Pagel
Helium and metal synthesis in massive stars at low $\mathrm{Z}$
F. Thielemann
Helium and metal abundances in HII regions
M. Grenon
C. Chiosi
SN and chemical synthesis for metal poor stars
The sites of nucleosynthesis: halo and bulge
Chemical evolution in the early Galaxy

The above contributions covering the major aspects of early nucleosynthesis in galaxies were delivered in a full day meeting. 